\title{
Competências de matemática e de literacia emergente: Estudo correlacional
}

\author{
Joana Amarali, Joana Cruzii, Patrícia Constanteiii, Patrícia \\ Pintoiv, Marta Almeidav, Elisa Lopesvi, Cristiana Silvavii, \\ Ana Macedoviii, Liliana Monteiroix, Teresa Oliveirax \& \\ Filipa Cruz ${ }^{\mathrm{xi}}$
}

Câmara Municipal de Matosinhos, Portugal

Resumo

Tem sido amplamente relatada a importância da implementação de projetos de intervenção que facilitem o desenvolvimento de competências de linguagem oral, consciência fonológica, linguagem escrita, e matemáticas, desde a educação pré-escolar, bem como a necessidade de identificação atempada de crianças em risco educacional. Este estudo apresenta como objetivo analisar em que medida o desempenho das crianças em idade préescolar nas competências de linguagem oral, consciência fonológica e linguagem escrita está correlacionado com o desenvolvimento de competências matemáticas. Participaram no estudo 99 crianças que frequentavam a educação pré-escolar. Recorreu-se a análises correlacionais de modo a perceber a relação entre as variáveis em questão. Os resultados permitem constatar que as competências em estudo estão correlacionadas entre si, de modo estatisticamente significativo, não sendo encontradas diferenças estatisticamente significativas entre rapazes e raparigas. Estes resultados sugerem a relevância do contexto de proveniência das crianças, apresentando implicações para a avaliação psicológica e para a prática pedagógica.

Palavras-chave

Competências matemáticas; Educação pré-escolar; Linguagem oral; Literacia emergente 


\section{Introdução}

A educação pré-escolar é atualmente encarada nacional e internacionalmente como um contexto privilegiado de promoção de competências facilitadoras da aprendizagem da leitura, da escrita e da matemática (Cadima et al., 2008; Justice \& Kaderavek, 2002; Justice, Weber, Ezell, \& Bakeman, 2002; Whitehurst \& Lonigan, 1998). A investigação tem evidenciado a natureza protetora e facilitadora da educação pré-escolar para esbater o designado 'efeito Mateus' (Stanovich, 1986), de acordo com o qual o modo como as crianças iniciam a escolaridade formal influencia o seu percurso escolar. Assim, os bons alunos tendem a construir percursos de sucesso e os alunos com dificuldades tendem a manter este padrão ao longo da escolaridade. Deste modo, o desempenho na leitura ao longo dos primeiros anos de escolaridade parece ser condicionado pelo tipo de abordagem efetuada à leitura e à escrita na educação pré-escolar (Pinto, 2012).

Os estudos mostram que este efeito também se verifica no que diz respeito às competências matemáticas, uma vez que, embora seja possível reduzir diferenças individuais através de programas de intervenção, as crianças com pior desempenho tendem a mantê-lo ao longo do tempo (Aunola, Leskinen, Lerkkanen, \& Nurmi, 2004; Cruz, Amaral, Almeida, \& Constante, 2014; Ginsburg, Lee, \& Boyd, 2008; Purpura, Baroody, \& Lonigan, 2013). Neste sentido, é fundamental que se criem as melhores condições para que as crianças iniciem a escolaridade formal bem preparadas para as exigências futuras. Estas evidências são ainda mais relevantes quando se abrangem crianças de contextos desfavorecidos, nomeadamente em termos culturais, pois, para além da reduzida acessibilidade a materiais de leitura, escrita e matemática e da existência de experiências reduzidas de contacto com a linguagem escrita e com a matemática, apresentam, por vezes, um frágil domínio de competências consideradas preditoras das aprendizagens formais nestes domínios (Duursma, Augustyn, \& Zuckerman, 2008; Jordan, Glutting, Ramineni, \& Watkins, 2010).

O jardim de infância assume-se, assim, como um espaço importante na criação de oportunidades e experiências significativas de qualidade desenvolvimental, que propiciam a construção de conhecimentos facilitadores das posteriores aprendizagens formais, e na discriminação positiva das 
crianças provenientes de meios desfavorecidos, procurando colmatar as desvantagens sociais e estimulando as diferentes áreas do seu desenvolvimento (Cruz, Oliveira, et al., 2014; Duursma et al., 2008; Viana \& Martins, 2009). As principais aprendizagens realizadas pelas crianças na educação pré-escolar decorrem da brincadeira, já que é incentivado o "aprender a brincar". As atividades lúdicas fazem parte do quotidiano dos jardins de infância e são mais apelativas e consideradas apropriadas, uma vez que facilitam a realização de interações positivas entre os adultos e as crianças (Dionísio \& Pereira, 2006; Mata, 2008; Sonnenschein \& Munsterman, 2002). Já em 1984, Bruner salientava a importância das brincadeiras e do jogo infantil para a exploração, realçando que a aprendizagem da língua materna é facilitada quando se inscreve no campo lúdico.

São bem descritas na literatura as competências consideradas preditoras de sucesso no domínio da leitura e da escrita, designadamente as relacionadas com a linguagem oral (vocabulário, conhecimentos morfossintáticos, memória auditiva para material verbal), a consciência fonológica (especificamente, a consciência silábica e fonémica) e os conhecimentos concetuais (aspetos figurativos e convencionais da linguagem escrita) e processuais (por exemplo, nome das letras) relacionados com a linguagem escrita (Duursma et al., 2008; Ferreiro \& Teberosky, 1984; Lopes, 2010; Martins \& Niza, 1998; Stephenson, Parrila, Georgiou, \& Kirby, 2008; Viana, 2005; Yopp \& Stapleton, 2008). À medida que as crianças contactam com a linguagem escrita desenvolvem não só competências e conhecimentos relacionados com o vocabulário, o conhecimento sintático, a consciência fonológica, a funcionalidade e direcionalidade da escrita, mas também um conjunto de atitudes e emoções face à linguagem escrita. A dimensão motivacional apresenta-se igualmente importante para a construção de projetos de leitores/escritores e para o sucesso escolar, ou seja, para a construção de sentidos e de razões para a aprendizagem da leitura e da escrita (Bamberger, 1975; Martins \& Niza, 1998; Mata, 2008).

No domínio da matemática, a investigação sugere que o conhecimento matemático surge como uma atividade cognitiva informal (Ginsburg, 1989), que a criança adquire de forma espontânea e a partir das vivências quotidianas e de atividades lúdicas (Cadima et al., 2008). Têm sido apontadas como competências basilares e preditoras de sucesso escolar neste domínio 
a contagem, competência esta central no desenvolvimento do conhecimento do número e presumivelmente uma das atividades mais comuns realizadas em casa e na escola, a relação entre os números e a construção do sentido de número, a cardinalidade, a conexão dos símbolos escritos a quantidades distintas e as operações simples, como adições e subtrações, os padrões, as formas geométricas e as medições (Cadima et al., 2008; Greenes, Ginsburg, \& Balfanz, 2004; Jordan et al., 2010; Purpura et al., 2013).

Paralelamente ao estudo sobre o impacto de intervenções de promoção das competências consideradas preditoras de sucesso nos domínios da leitura, escrita e matemática, a investigação realça a pertinência da identificação atempada das crianças em risco educacional (Jordan et al., 2010; Purpura et al., 2013). O desempenho diferencial dos alunos em função do sexo tem igualmente sido considerado como uma preocupação das investigações (Below, Skinner, Fearrington, \& Sorrel, 2010; Matthews, Ponitz, \& Morrison, 2009; Mazzocco \& Thompson, 2005; Skaalvik \& Rankin, 1994). No âmbito da aprendizagem formal da leitura e da escrita, tende a ser consensual a existência de uma vantagem das raparigas (Below et al., 2010). No âmbito da matemática, os resultados são ambíguos (Mazzocco \& Thompson, 2005; Skaalvik \& Rankin, 1994). Na educação pré-escolar, ainda sem ocorrer uma aprendizagem formal, os estudos não são conclusivos sobre a influência do sexo no desempenho das crianças (Matthews et al., 2009; Mazzocco \& Thompson, 2005).

O presente estudo apresenta como objetivo central analisar em que medida o desempenho das crianças em idade pré-escolar nas competências de linguagem oral, consciência fonológica e linguagem escrita está correlacionado com o desenvolvimento de competências matemáticas. Deste modo, pretende-se perceber em que medida o desenvolvimento destas competências é harmonioso, correlacional e dependente das oportunidades proporcionadas às crianças, aquando da entrada na educação pré-escolar. Pretende-se ainda analisar de que modo o desempenho das crianças, nestas idades, é diferente em função do sexo. 


\section{Método}

\section{Participantes}

Participaram neste estudo 99 crianças que frequentavam a educação pré-escolar em dois agrupamentos de escolas da rede pública do concelho de Matosinhos. O concelho de Matosinhos pertence à Área Metropolitana do Porto e tem vindo a aumentar a sua visibilidade em termos educacionais e culturais. Neste concelho, de acordo com os Censos 2011, habitam 175.478 indivíduos, tendo-se assistido, na última década, a um aumento da sua população residente, na ordem dos $5 \%$. A rede escolar, no concelho de Matosinhos, engloba as diversas tipologias de estabelecimentos de educação e ensino público, privado e cooperativo que desenvolvem a sua atividade abrangendo a Educação Pré-Escolar, o Ensino Básico, o Ensino Secundário, o Ensino Profissional, o Ensino Superior e as Universidades Seniores.

Os participantes do presente estudo eram provenientes de sete jardins de infância, correspondendo a um total de 14 educadoras de infância. Apenas três destas educadoras não tinham vínculo estável na carreira. Trata-se de uma amostra de conveniência relacionada com a implementação de projetos autárquicos de promoção de competências facilitadoras do sucesso escolar, no âmbito da linguagem oral, da linguagem escrita e da matemática, cuja intervenção tem início aos 4 anos de idade.

O estudo realizou-se no início do ano letivo 2013/14 (entre outubro e novembro) e a média de idades das crianças era, na altura da avaliação, de 3.88 anos (DP $=3.28$ ). As crianças estavam distribuídas de modo equitativo relativamente ao sexo: $51.5 \%$ das crianças eram do sexo masculino $(N=51)$ e $47.5 \%$ do sexo feminino $(N=48)$. Não pertencem a esta amostra crianças elegíveis para a Educação Especial.

De acordo com os dados dos projetos educativos dos agrupamentos de escolas, os pais das crianças trabalhavam predominantemente nos setores secundário e terciário. Três dos jardins de infância de proveniência das crianças situavam-se em meios socioculturais desfavorecidos e os restantes quatro em zonas culturalmente mais favorecidas. As crianças eram maioritariamente oriundas de famílias de classe média e média baixa, registando-se quer situações de desemprego, quer de emprego precário. A maioria das mães possuía habilitações literárias entre o $9 .^{\circ}$ e $012 .^{\circ}$ anos, embora cerca de $25 \%$ já tivessem uma licenciatura. As crianças tinham 
ingressado pela primeira vez nas salas de 4 anos dos jardins de infância alvo de estudo.

\section{Instrumentos}

Para a avaliação da linguagem oral foram utilizados os seguintes instrumentos:

- Nomeação (Sim-Sim, 2004): incluída na bateria de provas de avaliação da linguagem oral (Sim-Sim, 2004), esta prova pode ser aplicada a crianças entre os 4 e os 9 anos e avalia o conhecimento lexical; tem uma pontuação máxima de 70 pontos e apresenta um valor de consistência interna considerado elevado $(a=.89)$.

- Compreensão de Estruturas Complexas (Sim-Sim, 2004): incluída na bateria de provas de avaliação da linguagem oral (Sim-Sim, 2004), esta prova pode ser aplicada a crianças entre os 4 e os 9 anos e avalia as capacidades recetivas da criança no domínio linguístico sintático; tem uma pontuação máxima de 32 pontos e apresenta um valor de consistência interna considerado elevado ( $a$ $=.90)$.

Para a avaliação do processamento fonológico foi utilizada a prova:

- Classificação da Sílaba Inicial (Silva, 2003): subteste da Bateria de Provas Fonológicas (Silva, 2003), que se destina a crianças em idade pré-escolar (dos 5 aos 6 anos e 4 meses) e que avalia as competências fonológicas infantis. Esta prova consiste em 14 itens e avalia a capacidade da criança detetar sílabas iniciais idênticas em diferentes palavras. Apresenta um valor de consistência interna considerado elevado ( $a=.77)$.

Para a avaliação das concetualizações infantis sobre a linguagem escrita, foram utilizados os seguintes instrumentos:

- Reconhecimento de letras maiúsculas: esta prova avalia o conhecimento sobre o nome das letras maiúsculas. As letras do alfabeto, escritas em letra maiúscula, são apresentadas sucessivamente de modo aleatório, em formato digital, e é solicitado à criança que diga qual a letra que surge no ecrã. A pontuação máxima nesta prova é de 26 pontos. 
- Reconhecimento de letras minúsculas: procedimento idêntico ao anterior, em que se apresentaram, de modo digital, as letras minúsculas. A pontuação máxima nesta prova é de 26 pontos.

- Escrita de pseudopalavras: esta prova tem como objetivo avaliar as concetualizações da criança sobre a linguagem escrita. São ditadas dez pseudopalavras e é pedido à criança que as escreva. As respostas são cotadas segundo o sistema de classificação fónico (Mann, 1993). Cada resposta pode receber entre zero e quatro/cinco pontos (de acordo com o número de letras existente na palavra). São atribuídos zero pontos no caso de não ser escrita qualquer letra ou no caso de as letras não representarem nenhum aspeto da estutura fonológica da palavra. O total máximo de pontos em cada palavra é atribuído no caso de a palavra ser escrita de modo fonologicamente correto. A pontuação máxima é de 40 pontos.

- Tema-3. Test of Early Mathematic Achievement (Ginsburg \& Baroody, 2003): este instrumento tem o objetivo de avaliar o conhecimento matemático formal e informal de crianças entre os 3 anos e zero meses e os 8 anos e 11 meses de idade. Este teste é composto por 72 itens, organizados por ordem de dificuldade e por faixa etária. Foi efetuada uma tradução para a população portuguesa por Cadima e colaboradores (2008), sendo analisadas as caraterísticas psicométricas do instrumento, que apresenta coeficientes de consistência interna superiores a .90 .

\section{Procedimentos}

Para a realização do estudo foi solicitada a colaboração de dois agrupamentos de escolas do concelho de Matosinhos, nos quais estão a ser desenvolvidos projetos autárquicos de promoção do sucesso escolar desde a educação pré-escolar. Na reunião ordinária de início de ano letivo, efetuada com os pais das crianças das salas de 4 anos, foram-lhes apresentados os projetos e foi-lhes distribuída a folha de autorização, que incluía a avaliação das competências de linguagem oral, consciência fonológica, linguagem escrita e competências matemáticas. 
A avaliação das crianças ocorreu no primeiro período do ano letivo 2013/14, quando as crianças ingressaram nos jardins de infância (setembronovembro). A avaliação das crianças foi efetuada de modo individual. Cada avaliação tinha a duração aproximada de 50 minutos. A ordem de aplicação das provas foi igual para todas as crianças. A sua avaliação decorreu no jardim de infância durante a componente letiva.

\section{Análises estatísticas}

As análises estatísticas foram efetuadas com recurso ao software IBM ${ }^{\circledR}$ SPSS ${ }^{\circledR} 20$. Foi realizada a análise da estatística descritiva (medidas de tendência central e dispersão) relativa aos resultados obtidos nas provas utilizadas na avaliação da literacia emergente e das competências matemáticas.

Posteriormente, foi analisado o pressuposto da normalidade das distribuições para cada variável, através do teste Kolmogorov-Smirnov. De acordo com a normalidade das mesmas, realizou-se a estatística bivariada, com recurso quer à correlação de Spearman, quer à correlação de Pearson. Foi ainda comparada a diferença de desempenhos nas diferentes provas em função do sexo das crianças, através do teste t de Student para amostras independentes e do teste não paramétrico Mann-Whitney.

\section{Resultados}

No quadro 1 são apresentados os desempenhos médios das crianças em cada prova avaliada.

Quadro 1 - Resultados globais das provas

\begin{tabular}{llllll}
\hline Prova & Média & DP & Amp. & Assimetria & Curtose \\
\hline Nomeação & 31.96 & 10.74 & $6.00-56.00$ & -.40 & .03 \\
Compreensão Estruturas Complexas & 11.61 & 5.63 & $.00-23.00$ & -.07 & -.51 \\
Classificação Sílaba Inicial & .85 & 1.52 & $.00-5.00$ & 1.66 & 1.43 \\
Letras Maiúsculas & 3.90 & 5.67 & $.00-26.00$ & 1.86 & 2.99 \\
Letras Minúsculas & 2.09 & 3.40 & $.00-24.00$ & 3.61 & 18.24 \\
Escrita Pseudopalavras & .33 & 1.59 & $.00-10.00$ & 5.05 & 25.42 \\
Tema-3 & 11.48 & 6.15 & $.00-28.00$ & 1.79 & -.49 \\
\hline
\end{tabular}


A estatística descritiva permite analisar o desvio-padrão das diferentes variáveis, verificando-se uma elevada dispersão dos desempenhos, em cada prova.

A análise da estatística descritiva permite constatar que a maioria das crianças apresenta desempenhos esperados para a sua idade. É ainda possível verificar a existência de um desempenho médio superior ao esperado para a idade na prova de nomeação. Relativamente ao conhecimento matemático, o quadro 1 permite verificar que a maioria das crianças possui conhecimentos matemáticos basilares, encontrando-se ligeiramente abaixo dos valores esperados para a idade. Os resultados médios nesta prova sugerem que as crianças apresentam competências elementares, como a contagem e a cardinalidade.

A análise da normalidade das distribuições, através do teste Kolmogorov-Smirnov, permitiu verificar que as variáveis Tema-3 e Compreensão de Estruturas Complexas apresentavam uma distribuição normal ( $p>$.05), enquanto as variáveis Nomeação, Classificação da Sílaba Inicial, Letras Maiúsculas, Letras Minúsculas e Escrita de Pseudopalavras indicavam a não verificação do pressuposto da normalidade $(p<.05)$. Face a estes resultados, foram realizadas correlações de Spearman e de Pearson para analisar a relação entre as variáveis em estudo.

\section{Quadro 2 - Análises de correlação entre as variáveis}

\begin{tabular}{|c|c|c|c|c|c|c|c|}
\hline & Nomeação & Compreensão & $\begin{array}{l}\text { Classificação } \\
\text { Sílaba Inicial }\end{array}$ & $\begin{array}{c}\text { L. } \\
\text { Maiúsculas }\end{array}$ & $\begin{array}{c}\text { L. } \\
\text { Minusculas }\end{array}$ & Escrita & Tema-3 \\
\hline Nomeação & 1 & & & & & & \\
\hline Compreensão & $.617^{* *}$ & 1 & & & & & \\
\hline $\begin{array}{l}\text { Classificação Sílaba } \\
\text { Inicial }\end{array}$ & $.516^{\star \star}$ & $.485^{\star \star}$ & 1 & & & & \\
\hline Letras Maiúsculas & $.463^{\star *}$ & $.311^{*}$ & $.381^{\star \star}$ & 1 & & & \\
\hline Letras Minúsculas & $.456^{* *}$ & $.304^{\star}$ & $.394^{\star \star}$ & $.941^{* *}$ & 1 & & \\
\hline Escrita & .138 (n.s.) & $.305^{\star}$ & $.299^{\star}$ & $.303^{\star}$ & $.302^{\star}$ & 1 & \\
\hline Tema-3 & $.573^{\star \star *}$ & $.548^{\star \star}$ & $.445^{\star \star}$ & $.634^{\star *}$ & $.630^{\star \star \star}$ & $.305^{\star}$ & 1 \\
\hline
\end{tabular}

A análise do quadro 2 evidencia a existência de relações positivas e estatisticamente significativas entre todas as variáveis em estudo, exceto entre a Nomeação e a Escrita de Pseudopalavras.

No que concerne às competências de linguagem oral e consciência fonológica, constata-se que apresentam correlações fortes, iguais ou 
superiores a $r=.49$. As concetualizações das crianças sobre a linguagem escrita apresentam correlações mais baixas, mas estatisticamente significativas, com as competências de linguagem oral e consciência fonológica, oscilando entre .30 e .46 .

As concetualizações sobre a linguagem escrita encontram-se igualmente correlacionadas de modo estatisticamente significativo entre si, constatando-se que a correlação entre o conhecimento de letras maiúsculas e minúsculas é muito elevada $(r=.94, p<.001)$.

As correlações entre as competências matemáticas, avaliadas através do Tema-3, e as competências de linguagem oral, consciência fonológica e concetualizações sobre a linguagem escrita são todas positivas e estatisticamente significativas. As correlações mais robustas verificam-se entre o Tema-3 e o conhecimento de letras minúsculas $(r=.63, p<.001)$ e letras maiúsculas $(r=.63, p<.001)$, e as correlações mais baixas são encontradas entre o Tema-3 e a escrita de pseudopalavras $(r=.31, p<.01)$.

Para verificar as diferenças de desempenhos nas provas em função do sexo efetuaram-se testes t-Student para amostras independentes e recorreuse à estatística não paramétrica (Teste de Mann-Whitney), quando as variáveis não se distribuíam de acordo com a curva normal.

\section{Quadro 3 - Desempenhos nas provas em função do sexo}

\begin{tabular}{|c|c|c|c|c|c|}
\hline & \multicolumn{2}{|c|}{ Rapazes } & \multicolumn{2}{|c|}{ Raparigas } & \multirow{2}{*}{ Teste } \\
\hline & Média & DP & Média & DP & \\
\hline Nomeação & 30.31 & 9.93 & 33.71 & 11.39 & $U=994.50 ; p>.05$ \\
\hline Compreensão & 10.73 & 5.66 & 12.54 & 5.51 & $t(97)=1.62 ; p>.05$ \\
\hline Classificação Sílaba Inicial & .71 & 1.40 & 1.00 & 1.64 & $U=994.50 ; p>.05$ \\
\hline Letras Maiúsculas & 2.75 & 4.07 & 5.13 & 6.81 & $\mathrm{U}=994.50 ; p>.05$ \\
\hline Letras Minúsculas & 1.59 & 2.10 & 2.63 & 4.35 & $\mathrm{U}=994.50 ; p>.05$ \\
\hline Escrita & .18 & 1.26 & .50 & 1.88 & $U=994.50 ; p>.05$ \\
\hline Tema-3 & 11.00 & 6.00 & 12.00 & 6.34 & $t(97)=.81 ; p>.05$ \\
\hline
\end{tabular}

Os resultados das diferenças de médias em função do sexo demonstram que não existem diferenças estatisticamente significativas no desempenho das crianças em função do sexo ( $p>05)$. 


\section{Discussão e implicações}

Neste estudo procurou analisar-se a correlação entre (a) competências de linguagem oral, consciência fonológica e linguagem escrita e (b) competências matemáticas em crianças em idade pré-escolar. O estudo acrescenta evidência empírica da existência destas correlações entre competências de matemática e de leitura e escrita, em idade pré-escolar.

A análise dos desvios-padrão encontrados sugere uma dispersão dos desempenhos das crianças, em cada prova. As crianças têm desempenhos diferentes em diferentes áreas, apesar de terem idades próximas. Considerando que no momento da avaliação das crianças ainda não tinha existido intervenção por parte da escola, a dispersão de desempenho pode estar relacionada com as oportunidades proporcionadas às crianças no seu contexto familiar ou nas redes sociais de apoio até aos 4 anos de idade. Esta informação é particularmente relevante quando se trata de meios desfavorecidos, onde geralmente está presente uma menor estimulação das competências relacionadas com linguagem oral, consciência fonológica, linguagem escrita e competências de matemática (Stipek \& Ryan, 1997).

A análise das médias dos desempenhos sugere igualmente que os participantes apresentavam uma maior estimulação da linguagem oral, designadamente do vocabulário, avaliado através da prova de nomeação. Estes resultados podem ser explicados pelo modo de aquisição e de desenvolvimento da linguagem oral, que é influenciada pelas experiências quotidianas, pela existência de modelos sociais e de interações em torno da linguagem oral. Já as restantes competências, nomeadamente relacionadas com a consciência fonológica, o conhecimento de letras, a relação entre a oralidade e a linguagem escrita e os conhecimentos matemáticos, parecem necessitar de algum tipo de instrução explícita e intencional, que tende a ocorrer nos anos finais da educação pré-escolar (Viana, Cruz, \& Cadime, 2014; Yopp \& Stapleton, 2008).

Os resultados do estudo demonstram a existência de correlações estatisticamente significativas e positivas entre as diferentes variáveis. Deste modo, constata-se que, quando se verifica uma diminuída estimulação numa área, esta parece comprometer as restantes dimensões avaliadas. Neste sentido, considera-se pertinente investir em projetos e programas que 
proporcionem as melhores e mais facilitadoras condições para que as crianças frequentem a educação pré-escolar com qualidade, para que posteriormente possam iniciar a escolaridade básica munidas de ferramentas basilares e preditoras de sucesso (Cruz, Amaral, et al., 2014). Alguns autores, como Linder, Ramey, e Zamback (2013) e McLoyd e Purtell (2008), sugerem que se as crianças entrarem no jardim de infância com lacunas ao nível das competências alvo do presente estudo, e que se estas não forem esbatidas desde cedo na infância, poderão manter-se ao longo do seu percurso escolar. Em contrapartida, quanto mais uma criança for estimulada numa determinada competência, melhor preparada se apresenta na sua aprendizagem formal. Estes resultados têm sido encontrados em diferentes investigações, salientando-se a existência de correlações estatisticamente significativas entre as concetualizações sobre a linguagem escrita na educação pré-escolar e o desempenho em leitura no final do $1^{\circ}$ ano de escolaridade (Alves Martins, 1996; Chauveau \& Rogovas-Chauveau, 2001).

Os resultados do estudo sugerem a ausência de correlação entre a escrita de pseudopalavras e a nomeação. Estes resultados podem estar relacionados com o facto de o vocabulário ser uma competência desenvolvida diariamente nos mais variados contextos. Por outro lado, a apropriação da linguagem escrita necessita de alguma forma de estimulação explícita e intencional, ou no jardim de infância, ou no contexto familiar (Cruz, 2011; Viana et al., 2014). A investigação tem evidenciado que a família tem um forte impacto no desenvolvimento da literacia das crianças, daí a relevância dos hábitos e dinâmicas familiares, não descurando a importância da educação pré-escolar (Martins, Mata, \& Silva, 2014). Uma reduzida estimulação na escrita poderá, assim, traduzir-se na inexistência de correlação com o vocabulário, devido a reduzida variabilidade na escrita de pseudopalavras.

Os resultados revelam ainda a presença de correlações significativas entre a linguagem oral e a consciência fonológica. Estes resultados têm igualmente sido encontrados em estudos nacionais e internacionais (Anthony et al., 2002; Coyne \& Harn, 2006), demonstrando a relevância da existência de atividades promotoras destas competências na educação pré-escolar.

Através dos resultados encontrados, é possível constatar a existência de correlações muito elevadas entre o conhecimento de letras maiúsculas e minúsculas. O facto de as correlações serem tão elevadas $(r=.941)$ sugere 
que, em estudos futuros, se possa apenas utilizar uma das variáveis. Estes dados sugerem ainda que, embora as crianças contactem predominantemente com letras impressas maiúsculas, desenvolvem igualmente conhecimentos sobre as letras impressas minúsculas (CardosoMartins \& Batista, 2005; Foulin, 2005; Viana et al., 2014). As concetualizações infantis sobre a escrita estão também correlacionadas com as variáveis de linguagem oral e consciência fonológica, ainda que com correlações mais baixas. Estes resultados salientam a apropriação, por parte das crianças, das relações entre a linguagem oral e a linguagem escrita, sendo também encontrados resultados idênticos noutros estudos (Martins \& Farinha, 2006; Silva, 2004).

Os resultados do presente estudo demonstram igualmente a existência de correlações positivas e estatisticamente significativas especificamente entre as competências matemáticas e as restantes variáveis, salientando a existência de correlações mais robustas entre o Tema-3 e o conhecimento de letras maiúsculas e minúsculas. Estes dados sugerem que o conhecimento do número parece estar relacionado com o conhecimento de letras, sendo competências que decorrem da existência de atividades intencionais e não do desenvolvimento ou ação do tempo. Resultados similares foram encontrados no $1^{\circ}$ ciclo do ensino básico (Hanline, Milton, \& Phelps, 2009).

Neste trabalho procurou-se ainda perceber se o desempenho nas diferentes provas era distinto em função do sexo. Os resultados encontrados demonstram a inexistência de diferenças entre sexos nos desempenhos nas competências de linguagem oral, consciência fonológica, linguagem escrita e competências matemáticas, à semelhança de outros estudos (Matthews et al., 2009; Mazzocco \& Thompson, 2005; Skaalvik \& Rankin, 1994). De um modo geral, estes resultados sugerem que os desempenhos diferenciados das crianças parecem estar mais relacionados com questões ambientais e contextuais do que com caraterísticas individuais ou desenvolvimentais.

Em termos de limitações, neste estudo recorreu-se ao Tema-3, que, embora seja a única prova aferida para a população portuguesa para avaliar conhecimentos matemáticos na educação pré-escolar, apresenta apenas um score total, não permitindo analisar o desempenho das crianças em competências parcelares, como a contagem, cardinalidade e operações. Constata-se ainda que o número reduzido de participantes pode colocar 
entraves à generalização dos resultados, pelo que, em estudos futuros, seria pertinente a aplicação da prova a um número maior de crianças. Finalmente, sugere-se que em trabalhos futuros se possa realizar um estudo similar e diferencial, em termos de nível socioeconómico.

De um modo geral, este estudo permitiu a análise conjunta de diferentes competências e a sua correlação. Os resultados encontrados apresentam implicações relevantes para a avaliação psicológica e para a prática pedagógica. Em termos de avaliação psicológica, sugere-se a necessidade de se atender a diferentes domínios do conhecimento das crianças, mesmo em idade pré-escolar, já que o modo como as crianças entram na escola deve ser considerado um ponto de chegada, e não um ponto de partida. Deste modo, será possível obter uma perspetiva global das competências consideradas preditoras de sucesso escolar. Esta avaliação deve apresentar como principal objetivo a identificação das crianças e das suas necessidades em termos de estimulação. Não se pretende que a avaliação psicológica se focalize apenas na testagem, nem na necessidade de "rotular" as crianças, mas antes na orientação da ação, ou seja, no conhecimento das idiossincrasias das crianças para a adequação das posteriores estratégias de intervenção (Gutkin, 2012).

Em termos de prática pedagógica, este estudo permite salientar a importância quer da identificação atempada das crianças, quer da intervenção sistemática e intencional na promoção do sucesso escolar desde o jardim de infância, de forma a esbater diferenças sociais e a acompanhar a resposta das crianças a intervenções deliberadas e empiricamente validadas (Kovaleski, 2007; Prewett et al., 2012).

O concelho de Matosinhos é pioneiro neste tipo de intervenções, estando atualmente no terreno dois projetos de promoção de competências facilitadoras da aprendizagem formal da leitura e da escrita - projeto "A Ler Vamos..." (dez anos de implementação) - e da matemática - "Matiga Matemática Amiga" (quatro anos de implementação). Ambos os projetos têm demonstrado validação empírica e constituem ferramentas de promoção da igualdade de oportunidades em termos de estimulação da linguagem oral, da linguagem escrita (Cruz et al., 2010; Cruz, Oliveira, et al., 2014; Cruz, Pinto, Pombal, \& Pinto, 2008) e das competências matemáticas (Cruz, Amaral, Almeida, \& Constante, 2014), através de uma intervenção lúdica, sistemática, 
intencional e não escolarizante. Ambos os projetos se baseiam na ação direta com as crianças, em contexto pré-escolar, privilegiando igualmente a modalidade indireta de intervenção, através da consultoria com docentes e encarregados de educação (Cruz, Pinto, Lopes, \& Pinto, 2015). Estes projetos incluem sessões em grande grupo, na sala, bem como intervenções em pequeno grupo, sendo o desempenho das crianças monitorizado ao longo dos dois últimos anos da educação pré-escolar. É privilegiada a prevenção e intervenção precoce, mais do que a remediação, e valorizado o empowerment dos técnicos e dos adultos que participam nos ambientes relevantes para as crianças, de forma a permitir a sustentabilidade das ações e a manutenção das mudanças que ocorrem durante a intervenção, já que, de acordo com a literatura, são estes agentes que facilitam o desenvolvimento de experiências enriquecedoras e o contacto com a leitura, a escrita e a matemática como algo inerente ao quotidiano das crianças (Abreu-Lima et al., 2012; Cruz et al., 2010; Pinto, 2012; Viana et al., 2014).

\section{Referências}

Abreu-Lima, I., Coelho, V., Lobo, C., Castro, C., Gomes, V., \& Monteiro, A. (2012). Promoção da competência matemática pré-escolar - Resultados de um programa de intervenção. In L. Mata, F. Peixoto, J. Morgado, J. Silva, \& V. Monteiro (Coords.), Actas do $12^{\circ}$ colóquio de Psicologia e Educação (pp 381394). Lisboa: ISPA.

Alves Martins, M. (1996). Pré-história de aprendizagem da leitura. Lisboa: ISPA.

Anthony, J. L., Lonigan, C. J., Burgess, S. R., Driscoll, L., Bacon, K., Phillips, B. M., \& Cantor, B. G. (2002). Structure of preschool phonological sensitivity: Overlapping sensitivity to rhyme, words, syllables, and phonemes. Journal of Experimental Child Psychology, 82, 65-92.

Aunola, K., Leskinen, E., Lerkkanen, M., \& Nurmi, J. (2004). Developmental dynamics of math performance from preschool to grade 2. Journal of Educational Psychology, 96, 699-713.

Bamberger, R. (1975). La promoción de la lectura. Paris: UNESCO.

Below, J. L., Skinner, C. H., Fearrington, J. Y., \& Sorrel, C. A. (2010). Gender differences in early literacy: Analysis of kindergarten through fifth-grade dynamic indicators of basic early literacy skills probes. School Psychology Review, 39(2), 240-257.

Bruner, J. (1984). Acción, pensamiento y lenguaje. Madrid: Alianza Editorial.

Cadima, S., Abreu-Lima, I., Gomes, V., Coelho, V., Lobo, C., \& Ramalho, C. (2008). Avaliação de competências de matemática dos 4 aos 7 anos de idade. In A. P. Noronha (Coord.), Actas da XIII Conferência Internacional de Avaliação 
Psicológica: Formas e Contextos (CD-ROM) (pp. 429-439). Braga: Psiquilibrios.

Cardoso-Martins, C., \& Batista, A. (2005). O conhecimento do nome das letras e o desenvolvimento da escrita: Evidência de crianças falantes de português. Psicologia: Reflexão e Crítica, 16(3), 491-502.

Chauveau, G., \& Rogovas-Chauveau, E. (2001). Des apprentis lecteurs en difficulté avant six ans. In G. Chauveau (Ed.), Comprendre l'enfant apprenti lecteur. Recherches actuelles en psychologie de l'écrit (pp. 32-43). Paris: Editions Retz.

Coyne, M., \& Harn, B. (2006). Promoting beginning reading success through meaningful assessment of early literacy skills. Psychology in the Schools, 43(1), 33-43.

Cruz, J. (2011). Práticas de literacia familiar e desenvolvimento literácito das crianças (Tese de doutoramento não publicada). Universidade do Minho, Braga.

Cruz, J., Amaral, J., Almeida, M., \& Constante, P. (2014). "MATIGA - Matemática Amiga": Um projeto de promoção de competências matemáticas na educação pré-escolar. Revista de Psicologia, Educação e Cultura, XVIII(1), 22-35.

Cruz, J., Costa, C., Silva, C., Silva, M., Pinto, P., Almeida, S., \& Santos, T. (2010). "A Ler Vamos...": Um projecto da Câmara Municipal de Matosinhos. In L. Salgado (Coord.), A educação de adultos: Uma dupla oportunidade na família (pp. 117126). Lisboa: Agência Nacional para a Qualificação, I.P.

Cruz, J., Oliveira, T., Almeida, M., Pinto, P., Constante, P., Lopes, E., ...Amaral, J. (2014). Projeto "A Ler Vamos..." - Um projeto autárquico de promoção da leitura. In F. Viana, R. Ramos, E. Coquet, \& M. Martins (Coords.), Atas do 10. ${ }^{\circ}$ Encontro Nacional (8. ${ }^{\circ}$ Internacional) de Investigação em Leitura, Literatura Infantil e llustração (CD-ROM, pp. 400-405) Braga: CIEC - Centro de Investigação em Estudos da Criança da Universidade do Minho.

Cruz, J., Pinto, P., Lopes, A., \& Pinto, A. C. (2015). O contributo das autarquias para a intervenção psicológica na educação infantil. Revista Amazônica, 8, XV(1),143160.

Cruz, J., Pinto, P., Pombal, F., \& Pinto, A. (2008). Práticas de promoção de competências pré-leitoras no concelho de Matosinhos. In P. Martins (Ed.), Actas do $1^{\circ}$ Congresso Internacional em Estudos da Criança. Infâncias Possíveis, Mundos Reais (CD-ROM) (pp. 1-9). Braga: Universidade do Minho.

Dionísio, M., \& Pereira, I. (2006). A educação pré-escolar em Portugal - Concepções oficiais, investigação e práticas. Perspectiva, 24, 597-622.

Duursma, E., Augustyn, M., \& Zuckerman, B. (2008). Reading aloud to children: The evidence. Archives of Disease in Childhood, 93(7), 554-557.

Ferreiro, E., \& Teberosky, A. (1984). Psicogénese da língua escrita. Porto Alegre: Artes Médicas.

Foulin, J. (2005). Why is letter-name knowledge such a good predictor of learning to read? Reading and writing, 18, 129-155.

Ginsburg, H. P. (1989). Children's arithmetic. Austin, Texas: Pro-Ed.

Ginsburg, H. P., \& Baroody, A. J. (2003). Test of early mathematic ability: Examiner's manual (3rd ed.). Austin Texas: Pro-Ed. 
Ginsburg, H. P., Lee, J. S., \& Boyd, J. S. (2008). Mathematics education for young children: What it is and how to promote it? Social Policy Report: Giving child and youth development knowledge away, 22, 3-22.

Greenes, C., Ginsburg, H. P., \& Balfanz, R. (2004). Big math for little kids. Early Childhood Research Quarterly, 19(1), 159-166.

Gutkin, T. (2012). Ecological psychology: Replacing the medical model paradigm for school based psychological and psychoeducational services. Journal of Educational and Psychological Consultation, 22, 1-20.

Hanline, M. F., Milton, S., \& Phelps, P. C. (2009). The relationship between preschool block play and reading and math abilities in early elementary school: A longitudinal study of children with and without disabilities. Early Child Development and Care, 180, 1005-1017.

Jordan, N. C., Glutting, J., Ramineni, C., \& Watkins, M. (2010).Validating a number sense screening tool for use in kindergarten and first grade: Prediction of mathematics proficiency in third grade. School Psychology Review, 39(2), 181195.

Justice, L. M., \& Kaderavek, J. (2002). Using shared storybook reading to promote emergent literacy. Teaching Exceptional Children, 34(4), 8-12.

Justice, L. M., Weber, S., Ezell, H., \& Bakeman, R. (2002). A sequential analysis of children's responsiveness to parental print references during shared book-reading interactions. American Journal of Speech-Language Pathology, 11, 30-40.

Kovaleski, J. (2007). Response to intervention: Considerations for research and systems change. School Psychology Review, 36(4), 638-646.

Linder, S. M., Ramey, M. D., \& Zambak, S. (2013). Predictors of success for school readiness: A selective review of the literature. Early Childhood Research and Practice, 15(1). Retrieved from http://ecrp.uiuc.edu/v15n1/linder.html

Lopes, J. (2010). Conceptualização, avaliação e intervenção nas dificuldades de aprendizagens: A sofisticada arquitectura de um equívoco. Braga: Psiquilibrios.

Mann, V. (1993). Phoneme awareness and future reading ability. Journal of Learning Disabilities, 4, 259-269.

Martins, M. A., \& Niza, I. (1998). Psicologia da aprendizagem da linguagem escrita. Lisboa: Universidade Aberta.

Martins, M. A., Mata, L., \& Silva, C. (2014). Conceptualizações sobre linguagem escrita - Percursos de investigação. Análise Psicológica, 2(XXXII), 135-143.

Martins, M., \& Farinha, S. (2006). Relação entre os conhecimentos iniciais sobre linguagem escrita e os resultados em leitura no final do $1^{\circ}$ ano de escolaridade. In C. Machado, L. Almeida, M. A. Guisande, M. Gonçalves, \& V. Ramalho (Coords.), Actas da XI Conferência Internacional de Avaliação Psicológica: Formas e Contextos (pp. 1051-1060). Braga: Psiquilibrios.

Mata, L. (2008). A descoberta da escrita. Textos de apoio para educadores de infância. Lisboa: Direcção-Geral de Inovação e de Desenvolvimento Curricular.

Matthews, J. S., Ponitz, C. C., \& Morrison, F. J. (2009). Early gender differences in selfregulation and academic achievement. Journal of Educational Psychology, 101(3), 689-704. 
Mazzocco, M. M., \& Thompson, R. E. (2005). Kindergarten predictors of math learning disability. Learning Disabilities Research \& Practice, 20, 142-155.

Mcloyd, V. C., \& Purtell, K. (2008). How childhood poverty and income affect children's cognitive functioning and school achievement. In S. Neuman (Ed.), Educating the other America: Top experts tackle poverty, literacy, and achievement in our schools (pp. 53-72). Baltimore: Paul H. Brookes.

Pinto, P. (2012). Experiências literácitas na educação pré-escolar e desempenho na leitura no $1 .^{\circ}$ e $2 .^{\circ}$ anos de escolaridade (Tese de doutoramento não publicada). Universidade do Minho, Braga.

Prewett, S., Mellard, D. F., Deshler, D. D., Allen, J., Alexander, R., \& Stern, A. (2012). Response to intervention in middle schools: Practices and outcomes. Learning Disabilities Research \& Practice, 27(3), 136-147.

Purpura, D., Baroody, A., \& Lonigan, C. (2013). The transition from informal to formal mathematical knowledge: Mediation to formal numeral knowledge. Journal of Educational Psychology, 105(2), 453-464.

Silva, A. C. (2003). Bateria de provas fonológicas. Lisboa: Relógio d' Água.

Silva, A. C. (2004). Descobrir o princípio alfabético. Análise Psicológica, 1(22), 187-191.

Sim-Sim, I. (2004). Avaliação da linguagem oral. Lisboa: Fundação Calouste Gulbenkian.

Skaalvik, E. M., \& Rankin, R. J. (1994). Gender differences in mathematics and verbal achievement, self-perception and motivation. British Journal of Educational Psychology, 64, 419-428.

Sonnenschein, S., \& Munsterman, K. (2002). The influence of home-based reading interactions on 5-years-olds' reading motivations and early literacy development. Early Childhood Research Quarterly, 17, 318-337.

Stanovich, K. (1986). Matthew effects in reading: Some consequences of individual differences in the acquisition of literacy. Reading Research Quarterly, 21, 360407.

Stephenson, K., Parrila, R., Georgiou, G., \& Kirby, J. (2008). Effects of home literacy, parents' beliefs and children's task-focused behavior on emergent literacy and word reading skills. Scientific Studies of Reading, 12(1), 24-50.

Stipek, R. S., \& Ryan, R. H. (1997). Economically disadvantaged preschoolers: Ready to learn but further to go. Developmental Psychology, 33, 711-723.

Viana, F. (2005). Avaliação e intervenção em dificuldades de aprendizagem da leitura. In M. C. Taveira (Org.), Psicologia escolar - Uma proposta científico-pedagógica (pp. 61-86). Coimbra: Quarteto.

Viana, F. L., Cruz, J., \& Cadime, I. (2014). "Ler" antes de ler. Como facilitar a aprendizagem da leitura e da escrita? In F. L. Viana \& I. Ribeiro (Coords.), Falar, ler e escrever. Propostas integradoras para jardim de infância. Carnaxide: Santillana.

Viana, F., \& Martins, M. (2009). Dos leitores que temos aos leitores que queremos. In I. Ribeiro \& F. Viana (Orgs.), Dos leitores que temos aos leitores que queremos. Ideias e projectos para promover a leitura (pp. 9-42). Coimbra: Almedina. 
Whitehurst, G. J., \& Lonigan, C. J. (1998). Child development and emergent literacy. Child Development, 69, 848-872.

Yopp, H., \& Stapleton, L. (2008). Conciencia fonémica en Español. The Reading Teacher, 61(5), 374-382. 


\title{
MATH SKILLS AND EMERGENT LITERACY: CORRELATIONAL STUDY
}

\begin{abstract}
It has been well documented the importance of the implementation of preschool interventions that facilitate the development of oral language, phonological awareness, written language and math skills, as well as the need of an early identification of children at educational risk. The aim of the present study is to examine the relationship between children's competence in oral language, phonological awareness, written language and mathematic competencies in preschool years. A total of 99 children participated in this study. Correlational analysis was used to understand the relation between the variables in the study. The results suggest that oral language, phonological awareness, written language and mathematic competencies are correlated. No statistical differences were found among boys and girls. These findings suggest that the growth in these skills may be related to contextual influences. These findings highlighted the implications to psychological evaluation and for pedagogic practice.
\end{abstract}

Keywords

Math skills; Kindergarten; Oral language; Emergent literacy

\section{COMPÉTENCES MATHÉMATIQUES ET LA LITTÉRATIE ÉMERGENTE: ÉTUDE CORRÉLATIONNELLE}

\section{Résumé}

Reconnaitre l'importance de la mise en œuvre de projets d'intervention qui facilitent le développement des compétences d'expression orale, de conscience phonologique, d'expression écrite et de compétences mathématiques, dès la maternelle, ainsi que la nécessité d'identifier en temps utile les enfants en risque éducationnel, le présent article vise analyser dans quelle mesure la performance des enfants en école maternelle au niveau du 
langage, de la conscience phonologique et de l'expression écrite sont liées au développement de leurs compétences mathématiques. Ont participé dans cette étude 99 enfants en école maternelle. Des analyses de corrélation ont été effectuées afin de comprendre la relation entre les variables en cause. Les résultats ont révélé que les compétences étudiées sont liées et il n'y avait pas de différences statistiques entre les sexes. Les résultats dévoilent également une influence de l'environnement social dont les enfants proviennent, ce qui présente des implications au niveau de l'évaluation psychologique ainsi que de la pratique pédagogique.

Mots-clé

Compétences mathématiques ; Éducation préscolaire ; Expression orale ; Littératie émergente

Recebido em outubro/2015 Aceite para publicação em julho/2016

i - xiDivisão de Educação, Câmara Municipal de Matosinhos, Portugal.

Toda a correspondência relativa a este artigo deve ser enviada para: Joana Cruz, Câmara Municipal de Matosinhos, Divisão de Educação, Avenida Dom Afonso Henriques, 4454-510 Matosinhos, Portugal. Email: joana.cruz@cm-matosinhos.pt 